\title{
Is Laparoscopic Power Morcellation of Fibroids a Cardinal Sin in 2017?
}

\author{
Gautam N. Allahbadia ${ }^{1}$
}

Received: 17 January 2017/Accepted: 17 January 2017/Published online: 30 January 2017

(C) Federation of Obstetric \& Gynecological Societies of India 2017

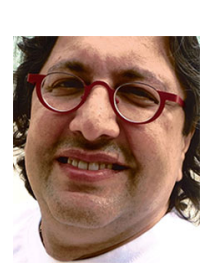

\begin{abstract}
About the Author
Gautam Nand Allahbadia MD is the Editor-in-Chief of the Journal of Obstetrics and Gynecology of India as well as the IVF Lite (Journal of Minimal Stimulation IVF). He is the Medical Director of Aster IVF and Women Clinic, Dubai, UAE as well as Rotunda-The Center for Human Reproduction, the world-renowned Infertility clinic at Bandra, Mumbai, India. He is a noted world authority on Ultrasound-guided Embryo Transfers and one of the pioneers in Third Party Reproduction in Southeast Asia. Dr. Allahbadia was responsible for India's first trans-ethnic Surrogate pregnancy involving a Chinese couple's baby delivered by an unrelated Indian surrogate mother. He cherishes over 150 peer-reviewed publications, 134 book chapters and 22 textbooks, the latest being a comprehensive text, entitled "Minimal Stimulation IVF," and is on the Editorial Board of several International Journals. Dr. Allahbadia has recently been elected as the Vice President of the World Association of Reproductive Medicine (WARM), headquartered in Rome, and "Mumbai's Top Doc" for 2012 by a peer nomination process.
\end{abstract} You can read more about his work at www.gautamallahbadia.com.

\begin{abstract}
The diagnosis of an unsuspected leiomyosarcoma after hysterectomy for the treatment of a presumed benign leiomyoma is a rare but highly clinically significant event. In order to facilitate removal of large uterine specimens using a minimally invasive surgical approach,

Gautam N Allahbadia, MD is the Editor-in-Chief of the Journal of Obstetrics and Gynecology of India as well as the IVF Lite (Journal of Minimal Stimulation IVF). He is the Medical Director of Aster IVF and Women Clinic, Dubai, UAE as well as Rotunda-The Center for Human Reproduction, Bandra, Mumbai, India.
\end{abstract}

\section{Gautam N. Allahbadia}

ivfwaladoc@gmail.com

1 Rotunda-The Center For Human Reproduction, Mumbai, India morcellation with extraction in pieces is often performed. In the event of unsuspected malignancy, this may result in abdominal dispersion of the tumor and contribute to poorer survival. Modern surgical innovations always work toward improving minimally invasive strategies. Laparoscopy, rooted in practices for years, supplanted laparotomy for many indications. For extraction of large uteri, morcellation is currently the only way to externalize surgical specimens (myomas, uteri), without increasing the skin opening while allowing to reduce postoperative complications when compared to laparotomy. However, in 2014, the Food and Drug Administration warned against the use of uterine morcellation because of an oncological risk. Some practicing academicians have challenged this recommendation. The incidence of uterine sarcomas is still poorly 
identified and preoperative diagnostic facilities remain inadequate. The small number of retrospective studies currently available do not reinforce any recommendation. The evaluation of morcellation devices and the improvement of preoperative diagnostic modalities (Imaging, preoperative Biopsy) are being improvised continually so as to minimize the oncological risks. Even during conventional myomectomy, tissue spillage occurs during resection of leiomyoma(s). Adverse oncologic outcomes of tissue morcellation should be mitigated through improved patient selection, preoperative investigations, and novel techniques that minimize tissue dispersion. Preoperative endometrial biopsy and cervical assessment to avoid morcellation of potentially detectable malignant and premalignant conditions is recommended.

\section{Introduction}

Mistakes you can learn from; sins stay with you forever. -Corey Taylor

Morcellation is the fragmentation of tissue to facilitate removal of the specimen through small incisions in minimally invasive surgery. This technique is not unique to gynecology and is used in general surgery as well, with the goal of improved surgical outcomes including decreased pain, cost, hospital length of stay, and rapid return to normal activities and work. Symptomatic fibroids are a common indication for hysterectomy or myomectomy. Although rare, unexpected gynecologic malignancies in presumed fibroids have been documented [1-3]. In cases where tissue retrieval is performed through morcellation, there is increasing concern that intra-abdominal dispersion of occult uterine malignancies may lead to peritoneal dissemination and worse outcomes [4]. Gynecologic laparoscopic power morcellation (LPM) has come under increased scrutiny over the last 2 years due to widespread attention to a known but rare complication, an unanticipated dissemination of malignancy, namely occult uterine leiomyosarcoma [5-7]. Three years ago, the Food and Drug Administration in the United States of America (FDA) issued the alert on power morcellation for uterine leiomyomas, addressing the risk of malignant cell spreading within the abdominal cavity (actual risk assessment from 1 in 360 to 1 in 7400 cases) [8]. The US Food and Drug Administration (FDA) warned against the use of laparoscopic power morcellators in the majority of women undergoing myomectomy or hysterectomy for the treatment of leiomyomas because of the concern for inadvertent spread of tumor cells if an undiagnosed cancer were to be present. The authors, representing a 46-member review group, reviewed the current literature to formulate prevalence rates of leiomyosarcoma in women with presumed leiomyomas and to assess reliable data regarding patient survival after morcellation [8]. They disagree with the FDA's methodology in reaching their conclusion and provide clinical recommendations for care of women with leiomyomas who are planning surgery [8].

The prevalence of occult leiomyosarcoma is debated; however, estimates from a robust meta-analysis suggest it may be in the range of 1 case per 1960-8300 fibroid surgeries [9]. Advancing age is an important clinical risk factor for occult malignancy. The impact of tumor morcellation may vary by mode of tissue removal, though tissue fragmentation is consistently associated with poorer outcomes. Decision and cost analyses continue to support laparoscopic hysterectomy as a low-morbidity and costeffective approach. The increased scrutiny on fibroid procedures in the past few years may lead to changes in surgical approach; however, alternative tissue extraction options are evolving, including incorporation of contained morcellation [9].

Extrauterine spread of leiomyomas is rare and most commonly occurs in the lungs. Increasing number of cases involving extrauterine spread of leiomyomas have been reported with the introduction of power morcellation. The exact pathogenesis is unknown but is likely multifactorial. Park et al. [10] presented a case of simultaneous metastatic leiomyomatosis to the lungs and peritoneal cavity following laparoscopic myomectomy with power morcellation. The patient presented to their institution for further management where she underwent a robotically assisted hysterectomy with bilateral salpingo-oophorectomy. Leiomyomatous implants measuring up to $2.4 \mathrm{~cm}$ were resected from bowel mesentery and bladder peritoneum. Subsequent serial computed tomography imaging confirmed stable pulmonary nodules without new intraperitoneal lesions. The authors emphasized that although the incidence of spread of benign disease is low, it is important to recognize this phenomenon as we will likely continue to encounter similar cases in the coming years [10].

Pieces of smooth muscle cell lost in the abdominal cavity during electrical morcellation after laparoscopic myomectomy may progress to leiomyomatosis peritonealis disseminata even after many years (10 years in this report) and it can be associated with ascites and lymph nodes enlargement [11].

Seventeen thousand nine hundred and three women underwent laparoscopic supracervical hysterectomy and 1603 underwent laparoscopic myomectomy in Perkin et al's study [12]. The rate of uterine cancer among women undergoing hysterectomy was 2.96 per 1000 and increased with age from 0 per 1000 at age younger than 35 to 9.07 per 1000 at ages 55-64 $(p<0.05$ for age $\geq 45$ compared with <45). Preoperative endometrial biopsy was 
documented in $47 \%$ of women subsequently diagnosed with uterine cancer. The overall rate of malignant and premalignant gynecological conditions was 5.14 per 1000 for laparoscopic supracervical hysterectomy and 1.87 per 1000 for laparoscopic myomectomy [12]. Approximately 1 in 350 women undergoing laparoscopic supracervical hysterectomy had an undiagnosed uterine cancer, with higher risks among older women. Approximately one-half of women had endometrial biopsies documented before hysterectomy. Safer alternatives, such as contained morcellation, should be evaluated to reduce the risk of complications after morcellation procedures [12].

\section{Discussion}

Raspagliesi et al. [13] investigated the impact of morcellation on survival outcomes of patients affected by undiagnosed uterine sarcoma. This was a retrospective study performed in 8 referral centers. Data of women undergoing morcellation for apparent benign uterine myomas who were ultimately diagnosed with stage I uterine sarcoma on final pathology were compared with data of women who did not undergo morcellation. Uterine sarcomas included: leiomyosarcomas (LMS), smooth muscle tumors of uncertain malignant potential (STUMP), low-grade endometrial stromal sarcomas (LG-ESS) and undifferentiated uterine sarcomas (UUS). Two-year survival outcomes were evaluated using Kaplan-Meier and Cox models. Overall 125 patients were identified: $31(24.8 \%), 21(16.8 \%)$ and $73(58.4 \%)$ patients had power morcellation during laparoscopy, non-power morcellation during open surgery and non-morcellation during open procedures, respectively. Considering patients affected by LMS, morcellation did not correlate with disease-free survival. However, patients undergoing either morcellation or power morcellation experienced a threefold increased risk of death in comparison with patients who had not morcellation. A trend toward an increase in recurrence was observed for patients undergoing morcellation for STUMP, while no differences in survival outcomes were observed for patients with LGESS and UUS. These data suggested that morcellation increases the risk of death in patients affected by undiagnosed LMS [13]. Further prospective studies are warranted in order to assess the risk-to-benefit ratio of power morcellator utilization in patients with apparent benign uterine myomas.

The aim of a recent study by Rechberger et al. [14] was to evaluate the rate of unexpected malignancies among women who underwent laparoscopic supracervical hysterectomy (LASH) with power morcellation. The retrospective analysis included clinical data of 426 consecutive female patients who underwent LASH with power morcellation due to presumed benign disorders $(78.4 \%$ symptomatic uterine fibromas, $12.7 \%$-abnormal uterine bleeding, $8.9 \%$-suspicion of uterine adenomyosis) between January 2011 and December 2015. Premalignant or malignant preoperative abnormalities in the cervix and the uterine corpus were contraindications for LASH. The unexpected malignancies were found in four patients from the study group: one ovarian cancer located on the inner part of simple ovarian cyst and 3 endometrial carcinomas $(0.9 \%)$ were documented. All these patients underwent abdominal reoperations and no histological abnormalities were detected in the extirpated cervix and adnexa. The study concluded that the incidence of unintended endometrial carcinoma in morcellated uteri after LASH was relatively small [14]. However, careful preoperative counseling should be undertaken in order to exclude the possibility of any malignant disease in uteri among women scheduled to power morcellation [14].

Rodriguez et al. [15] analyzed records of 13,964 women aged 25-64 years who underwent laparoscopic supracervical hysterectomies or myomectomies for leiomyomas from 2002 to 2011. Patient records were divided into two groups: history of laparoscopic supracervical hysterectomy and history of myomectomy. Subjects were tracked to identify diagnosis of leiomyosarcoma within 1 year of the procedure. They analyzed data from the 25-39, 40-49 and 50-64 age brackets. The results showed the incidence of occult leiomyosarcoma developing within 1 year following supracervical hysterectomy using a laparoscopic-assisted approach were $9.8,10.7$ and 33.4 per 10,000 for the $25-39$, 40-49 and 50-64 age brackets, respectively; the overall incidence rate was 13.1 per 10,000 . The incidence rate of occult leiomyosarcoma developing within 1 year following myomectomy using a laparoscopic-assisted approach were $0.0,33.8$ and 90.1 per 10,000 for the $25-39,40-49$ and 50-64 age brackets, respectively; the overall incidence rate was 17.3 per 10,000 . Their analysis showed the overall risk of being diagnosed with occult leiomyosarcoma was 12.9 per 10,000 in laparoscopic-assisted supracervical hysterectomy and myomectomy for patients younger than 49 [15]. There was no evidence of occult leiomyosarcoma 1 year after operation for patients younger than 40 who underwent laparoscopic myomectomy [15].

Iatrogenic parasitic myomas (PMs), caused by intra-corporeal power morcellation during laparoscopy, are gradually increasing. However, the pathogenesis and medical treatment of PMs remain largely unelucidated. Laparoscopically induced PM xenografted mouse model was conducted by xenografting human uterine myoma fragments into the abdominal cavity of SCID mice, and hormonal manipulation was performed using this mouse model to demonstrate the role of estrogen in the development of implanted PMs [16]. Immunohistochemistry of estrogen receptor $\alpha(\mathrm{ER} \alpha)$, 
progesterone receptor (PR), vimentin, vascular endothelial growth factor (VEGF), microvessel density (MVD) and Ki67 index were performed and compared. In the patient with $\mathrm{PMs}, \mathrm{ER} \alpha$ and PR, angiogenesis and proliferative property expression were upregulated in PM lesions compared to uterine myomas. In the laparoscopically induced PM mouse model, implanted myomas had more steroid receptor expressions, angiogenesis and proliferative property compared with prexenografted or non-implanted myoma. Depletion of estrogen in the ovariectomized (OVX) mice decreased laparoscopically induced PM implantations. In comparison, the implantations of PMs were increased with additional E2 supplement. Hormonal manipulation in the PM mouse model, including AI, GnRHa and SERM groups, were compared and AI significantly decreased the implantations, steroid receptor, angiogenesis, cell density and proliferative index of PMs compared with control group. Furthermore, GnRHa significantly decreased VEGF and MVD expressions compared with control group. These data highlight the crucial role of estrogen in the development of laparoscopically induced PMs and suggest that hormone manipulation may be a potential therapeutic agent [16].

Cases of parasitic leiomyoma involving prior laparoscopy were collected between 2012 and 2015 by Lu et al. [17] in a tertiary women's hospital in China. All six patients with parasitic leiomyoma had laparoscopic myomectomy or hysterectomy with power morcellation 39-132 months previously. Pathologically, these disseminated or parasitic leiomyomas did not show any evidence of malignancy. There were no morphological or immunohistochemical differences between the original tumor and the following seeding tumors.

Uncontained morcellation of leiomyomas during laparoscopic surgery has recently been discouraged, as undetected malignant tumors, namely leiomyosarcomas, could be fragmented which may result in upstaged disease [4]. However, enucleating leiomyomas per se may be inappropriate from an oncological perspective because complete, radical resection of malignant tumors to prevent further tumor growth or recurrence is not achieved. The aim of a study from the Netherlands was to determine whether spillage of leiomyoma cells occurs during open myomectomy [18]. Women undergoing open myomectomy were included in the study. Peritoneal abdominal washings were obtained on two occasions during the myomectomy procedure; the first one immediately after opening the abdomen and the second one after resection of the leiomyoma(s). Cytological evaluation of the fluids was performed to ascertain the presence of leiomyoma cells in any of the washings. Five patients were included in this pilot study. All first washings were negative for leiomyoma cells. However, cytology positive for the presence of leiomyoma cells was found in three of the five second, post-myomectomy washings. Tissue spillage from leiomyoma(s) occurs during conventional open myomectomy. The clinical relevance of tissue dissemination after myomectomy is unclear, but it cannot be excluded that this may negatively affect the patient's outcome if there is malignant change within the enucleated leiomyoma(s) [18]. Here, the authors questioned whether morcellation in specially designed containment bags after laparoscopic myomectomy guarantees any additional oncological safety [18].

Anapolski et al. [19] conducted a pilot study to obtain the first data concerning the safety of an endobag with three closable ports during morcellation and subsequent bag extraction under in vitro conditions, mimicking the settings in our operation theater. The second purpose of the study was to establish a minimal width of the skin incision necessary to safely extract the sealed bag after morcellation. The morcellation test was carried out on 11 stained porcine muscle tissue samples with one additional sample as a control. The insufflation pressure was set at $12 \mathrm{mmHg}$. After filling the endobag with blue dye solution, an additional extraction test was conducted by pulling the closed bag through a template with apertures of various diameters. For each opening, a series of ten bag extractions was carried out. No loss of solid material or fluid was recorded during the morcellation test. The extraction test showed a loss of fluid for template openings smaller than $18 \mathrm{~mm}$. The force necessary to extract the bag was inversely related to the width of the aperture. The data suggest that under the evaluated conditions, the use of a closable morcellation bag can considerably improve the patient's safety during morcellation [19]. Further studies are necessary to evaluate the influence of the bag on operating time, intervention costs and complications [19].

Ikhena et al. [20] set up a study to determine the feasibility and role of abdominopelvic washings at the time of laparoscopic power morcellation and to determine if endometrial or myometrial tissue will be detected before and after laparoscopic power morcellation. Abdominopelvic washings were performed before and after laparoscopic power morcellation in a specimen bag. Washings were evaluated for the presence of intra-abdominal endometrial or myometrial tissue using cell block and cytospin techniques. There was no visual or cytologic evidence of intra-abdominal dissemination of uterine tissue before or after enclosed morcellation on evaluation by cytospin or cell block techniques. Only the washings from the inside of the specimen bag were found to have myometrial tissue on evaluation using the cell block technique. When abdominopelvic washings are used as an intermediate outcome measure, enclosed bag morcellation appears to minimize tissue dissemination during laparoscopic power morcellation; however, additional and larger studies are needed [20]. 
The objective of Skorstad et al's [21] retrospective nationwide cohort study was to assess the operative treatment performed in women with uterine LMS in Norway in 2000-2012, including the number of morcellated LMS. The study participants were all women with histopathologically verified uterine LMS in Norway during 2000-2012. The data were collected from the Cancer Registry of Norway, National Patient Registry and medical records. There were 212 women diagnosed with uterine LMS in Norway in 2000-2012. Mean age at time of diagnosis was 58.1 years $(\mathrm{SD} \pm 12.5)$. The most frequent symptom in women suffering from LMS was abnormal uterine bleeding [110/212 (51.9\%)]. LMS was histopathologically verified in $49 / 212(23.1 \%)$ preoperatively. In $48 / 212(22.6 \%)$, a malignant condition was suspected and they were treated accordingly. In 115/212 (54.2\%), malignancy was not suspected at time of surgery and the women were treated according to the treatment protocol for fibroids. In only four patients was tissue retrieval by power morcellation conducted, accounting for $1.9 \%$ of all LMS cases. In more than $50 \%$ of women suffering from LMS, a malignant diagnosis was not confirmed or suspected prior to surgery. In this study, power morcellation of LMS has not lead to reduced survival. The authors suggest that power morcellators may be used in surgical treatment of selected cases of premenopausal women with symptomatic, presumed benign uterine leiomyomas [21].

The objective of Graebe et al's study was to determine the incidence of malignancies found in morcellated specimens at their institution [22]. Women who had a minimally invasive hysterectomy, for presumptive benign uterine conditions were identified, included and reviewed. Ten cases of malignancies were identified including endometrioid endometrial carcinomas [3], uterine serous carcinoma [1], endometrial stromal sarcomas (ESS) [3] and leiomyosarcomas (LMS) [3]. An overall risk of occult cancer on a morcellated specimen was $0.73 \%$, leiomyosarcoma was $0.22 \%$, endometrial stromal sarcoma $0.22 \%$ and endometrial cancer $0.29 \%$. The median uterine weight for the 10 morcellated malignancies was $293.5 \mathrm{~g}$, whereas the median weight for the benign uteri was only $117.5 \mathrm{~g}$. Morcellation was associated with substantially higher risk of abdominopelvic recurrence and lower disease-free survival [22]. Morcellated uterine malignancies were significantly heavier than benign uteri [22].

\section{Conclusions}

Although the incidence of occult leiomyosarcoma is low, outcomes are poor and may be worsened by morcellation [3-5]. By addressing risk factors for malignancy and incorporating evolving surgical techniques into practice, gynecologists can continue to offer patients a minimally invasive approach for fibroid management [4, 8, 23]. Studies published since the 2014 Food and Drug Administration safety communications offer updated leiomyosarcoma incidence estimates [8, 23, 24]. Incorporating these studies suggests that mortality rates are low following hysterectomy for presumed benign fibroids overall, and a minimally invasive approach remains a safe option [8, 23, 24]. Risk associated with morcellation, however, increases in women age $>50$ years due to increased leiomyosarcoma rates, an important finding for patient-centered discussions of treatment options for fibroids [23]. Hereditary cancer syndromes that increase the risk of uterine malignancy should be considered a contraindication to uncontained uterine morcellation [4]. Morcellation is an acceptable option for retrieval of benign uterine specimens and may facilitate a minimally invasive surgical approach, which is associated with decreased perioperative risks. Each patient should be counseled about the possible risks associated with the use of morcellation, including the risks associated with underlying malignancy [4]. Further research on uterine morcellation should focus on decision and cost-benefit analyses to determine the ideal candidate in whom uterine morcellation during minimally invasive hysterectomy would facilitate more good than harm [22].

Risk comes from not knowing what you are doing. -Warren Buffet

\section{References}

1. Parker W, Pritts E, Olive D. Risk of morcellation of uterine leiomyosarcomas in laparoscopic supracervical hysterectomy and laparoscopic myomectomy, a retrospective trial including 4791 women. J Minim Invasive Gynecol. 2015;22(4):696-7. doi: 10.1016/j.jmig.2015.01.015.

2. Tan-Kim J, Hartzell KA, Reinsch CS et al. Uterine sarcomas and parasitic myomas after laparoscopic hysterectomy with power morcellation. Am J Obstet Gynecol. 2015;212(5):594.e1-10. doi: 10.1016/j.ajog.2014.12.002.

3. Bogani G, Cliby WA, Aletti GD. Impact of morcellation on survival outcomes of patients with unexpected uterine leiomyosarcoma: a systematic review and meta-analysis. Gynecol Oncol. 2015;137(1):167-72. doi:10.1016/j.ygyno.2014.11.011.

4. Singh SS, Scott S, Bougie O et al. Technical update on tissue morcellation during gynaecologic surgery: its uses, complications, and risks of unsuspected malignancy. J Obstet Gynaecol Can. 2015;37(1):68-81.

5. Nugent W, Engelke G, Reicke S et al. Laparoscopic supracervical hysterectomy or myomectomy with power morcellation: risk of uterine leiomyosarcomas: a retrospective trial including 35.161 women in Germany. J Minim Invasive Gynecol. 2015;22(6S):S2-3. doi:10.1016/j.jmig.2015.08.013.

6. Nemec W, Inwald EC, Buchholz S et al. Effects of morcellation on long-term outcomes in patients with uterine leiomyosarcoma. Arch Gynecol Obstet. 2016;294(4):825-31. doi:10.1007/s00404-0164086-x. 
7. Zhang J, Li T, Zhang J et al. Clinical characteristics and prognosis of unexpected uterine sarcoma after hysterectomy for presumed myoma with and without transvaginal scalpel morcellation. Int J Gynecol Cancer. 2016;26(3):456-63. doi: 10.1097/IGC.0000000000000638.

8. Parker WH, Kaunitz AM, Pritts EA et al. Leiomyoma Morcellation Review Group US Food and Drug Administration's Guidance Regarding Morcellation of Leiomyomas: Well-Intentioned, But Is It Harmful for Women? Obstet Gynecol. 2016;127(1):18-22. doi:10.1097/AOG.0000000000001157.

9. Cohen SL, Hariton E, Afshar Y et al. Updates in uterine fibroid tissue extraction. Curr Opin Obstet Gynecol. 2016;28(4):277-82. doi:10.1097/GCO.0000000000000280.

10. Park BY, Leslie KO, Chen L et al. A case of simultaneous benign metastasizing leiomyomas and disseminated peritoneal leiomyomatosis following endoscopic power morcellation for uterine disease. Female Pelvic Med Reconstr Surg. 2017;23(1):e1-3.

11. Nappi L, Sorrentino F, Angioni S et al. Leiomyomatosis Peritonealis Disseminata (LPD) ten years after laparoscopic myomectomy associated with ascites and lymph nodes enlargement: a case report. Int J Surg Case Rep. 2016;25:1-3. doi: 10.1016/j.ijscr.2016.05.017.

12. Perkins RB, Handal-Orefice R, Hanchate AD et al. Risk of undetected cancer at the time of laparoscopic supracervical hysterectomy and laparoscopic myomectomy: implications for the use of power morcellation. Womens Health Issues. 2016;26(1):21-6. doi:10.1016/j.whi.2015.09.008.

13. Raspagliesi F, Maltese G, Bogani G et al. Morcellation worsens survival outcomes in patients with undiagnosed uterine leiomyosarcomas: A retrospective MITO group study. Gynecol Oncol. 2017;144(1):90-5. doi:10.1016/j.ygyno.2016.11.002.

14. Rechberger T, Miotła P, Futyma K et al. Power morcellation for women undergoing laparoscopic supracervical hysterectomysafety of procedure and clinical experience from 426 cases. Ginekol Pol. 2016;87(8):546-51. doi:10.5603/GP.2016.0042.

15. Rodriguez AM, Asoglu MR, Sak ME et al. Incidence of occult leiomyosarcoma in presumed morcellation cases: a database study. Eur J Obstet Gynecol Reprod Biol. 2016;197:31-5. doi: 10.1016/j.ejogrb.2015.11.009.

16. Huang BS, Yang $\mathrm{MH}$, Wang $\mathrm{PH}$ et al. Oestrogen-induced angiogenesis and implantation contribute to the development of parasitic myomas after laparoscopic morcellation. Reprod Biol Endocrinol. 2016;14(1):64.

17. Lu B, Xu J, Pan Z. Iatrogenic parasitic leiomyoma and leiomyomatosis peritonealis disseminata following uterine morcellation. J Obstet Gynaecol Res. 2016;42(8):990-9. doi:10.1111/jog.13011.

18. Sandberg EM, van den Haak L, Bosse $T$ et al. Disseminated leiomyoma cells can be identified following conventional myomectomy. BJOG. 2016;123(13):2183-7. doi:10.1111/1471-0528.14265.

19. Anapolski M, Panayotopoulos D, Alkatout I et al. Preclinical safety testing for morcellation and extraction for an endobag with sealable ports: in vitro pilot study. Surg Endosc. 2017;31(1):494-500. doi:10.1007/s00464-016-4969-8.

20. Ikhena DE, Paintal A, Milad MP. Feasibility of washings at the time of laparoscopic power morcellation: a pilot study. J Minim Invasive Gynecol. 2016;23(5):793-7. doi:10.1016/j.jmig.2016.03.024.

21. Skorstad M, Kent A, Lieng M. Uterine leiomyosarcoma-incidence, treatment, and the impact of morcellation: a nationwide cohort study. Acta Obstet Gynecol Scand. 2016;95(9):984-90. doi:10.1111/aogs.12930.

22. Graebe K, Garcia-Soto A, Aziz M et al. Incidental power morcellation of malignancy: a retrospective cohort study. Gynecol Oncol. 2015;136(2):274-7. doi:10.1016/j.ygyno.2014.11.018.

23. Siedhoff MT, Doll KM, Clarke-Pearson DL et al. Laparoscopic hysterectomy with morcellation vs abdominal hysterectomy for presumed fibroids: an updated decision analysis following the 2014 Food and Drug Administration safety communications. Am J Obstet Gynecol. 2016; d doi:10.1016/j.ajog.2016.11.1039.

24. Harris JA, Swenson CW, Uppal S et al. Practice patterns and postoperative complications before and after Food and Drug Administration safety communication on power morcellation. Am J Obstet Gynecol. 2015;. doi:10.1016/j.ajog.2015.08.047. 